\title{
Controlador de campo de corrente alternada para câmara de eletroformação de
}

\section{vesículas unilamelares gigantes}

\author{
Alternating current field controller for giant unilamellar vesicle electroformation chamber \\ Controlador de campo de corriente alterna para cámara de electroformación de vesículas \\ unilaminares gigantes
}

Recebido: 15/12/2021 | Revisado: 22/12/2021 | Aceito: 08/01/2022 | Publicado: 11/01/2022

\author{
Juracy Leandro dos Santos Junior \\ ORCID: https://orcid.org/0000-0003-4055-1889 \\ Universidade Federal de Goiás, Brasil \\ E-mail: juracyleandro@ufg.br \\ Sílvio Leão Vieira \\ ORCID: https://orcid.org/0000-0003-4523-0769 \\ Universidade Federal de Goiás, Brasil \\ E-mail: slvieira@ufg.br
}

\begin{abstract}
Resumo
A produção de vesículas unilamelares gigantes, GUV tem sido objeto de muitos estudos devido à sua simplicidade e por mimetizar funções complexas essenciais das membranas biológicas. A produção de GUVs é obtida por meio da exposição de filmes lipídicos a uma solução aquosa na presença de campos elétricos aplicados entre dois eletrodos à temperatura estável, processo chamado de eletroformação. O controle da amplitude de campo dos eletrodos imersos na solução que ocorre o processo de eletroformação é de extrema importância para eficiência da formação das GUVs a serem utilizadas em protocolos de treinamento de análises para aplicações biomédicas. O objetivo deste trabalho será avaliar um método de controle automático da estabilidade da amplitude do campo elétrico em solução aquosa. Foi desenvolvido um sistema de controle baseado em um atenuador e amplificador controlado por tensão, VCA que usa modulação por largura de pulso, PWM para controlar a amplitude do campo elétrico. A estabilidade da amplitude do campo ao longo do tempo foi avaliada por meio de um campo elétrico aplicado com frequência de $500 \mathrm{~Hz}$ e amplitude de $600 \mathrm{mV}$ RMS. O protótipo do circuito de controle VCA foi testado como atuador de um algoritmo de controle proporcional-integral e derivativo, PID para um sistema de controle em malha fechada da amplitude do campo aplicado entre dois eletrodos. Seu desempenho foi avaliado em soluções salinas e em água pura. Os valores médios do campo ao longo do tempo com controle PID apresenta tendência de queda em relação ao valor inicial de $0,3 \%$. O circuito de controle VCA proposto mostrou-se eficaz na manutenção dos valores dos campos ao longo do tempo.
\end{abstract}

Palavras-chave: Amplificador controlado por tensão; Controlador derivado integral proporcional; Campo elétrico alternado; Eletroformação; Vesículas unilamelares gigantes.

\begin{abstract}
The production of giant unilamellar vesicles, GUV has been the subject of many studies due to its simplicity and for mimicking essential complex functions of biological membranes. The production of GUVs is obtained by exposing lipid films to an aqueous solution in electric fields applied between two electrodes at a stable temperature, a process called electroformation. Controlling the field amplitude of electrodes immersed in the solution in the electroformation process is extremely important for the efficiency of the formation of GUVs to be used in training protocols for analysis for biomedical applications. The objective of this work will be to evaluate an automatic control method for the stability of the electric field amplitude in an aqueous solution. A control system based on a voltage-controlled attenuator and amplifier, VCA that uses pulse-width modulation, PWM to control the amplitude of the electric field was developed. The stability of the field amplitude over time was evaluated using an applied electric field with a frequency of $500 \mathrm{~Hz}$ and an amplitude of $600 \mathrm{mV}$ RMS. The VCA control circuit prototype was tested as an actuator of a proportionalintegral and derivative, PID control algorithm for a closed-loop control system of the applied field amplitude between two electrodes. Its performance was evaluated in saline solutions and pure water. The mean values of the field over time with PID control show a tendency to fall from the initial value of $0.3 \%$. The proposed VCA control circuit proved to maintain field values over time effectively.
\end{abstract}

Keywords: Voltage-controlled amplifier; Proportional-integral derivative controller; Alternating electric field; Electroformation; Giant unilamellar vesicles. 


\begin{abstract}
Resumen
La producción de vesículas unilaminares gigantes, GUV ha sido objeto de muchos estudios debido a su simplicidad y por imitar funciones complejas esenciales de las membranas biológicas. La producción de GUV se obtiene exponiendo películas lipídicas a una solución acuosa en presencia de campos eléctricos aplicados entre dos electrodos a una temperatura estable, proceso llamado electroformación. Controlar la amplitud de campo de los electrodos sumergidos en la solución que tiene lugar en el proceso de electroformación es de suma importancia para la eficiencia de la formación de GUV que se utilizarán en protocolos de entrenamiento para análisis para aplicaciones biomédicas. El objetivo de este trabajo será evaluar un método de control automático para la estabilidad de la amplitud del campo eléctrico en solución acuosa. Se desarrolló un sistema de control basado en un atenuador y amplificador controlado por voltaje, VCA que usa modulación de ancho de pulso, PWM para controlar la amplitud del campo eléctrico. La estabilidad de la amplitud del campo a lo largo del tiempo se evaluó utilizando un campo eléctrico aplicado con una frecuencia de $500 \mathrm{~Hz}$ y una amplitud de $600 \mathrm{mV}$ RMS. El prototipo del circuito de control VCA se probó como un actuador de un algoritmo de control proporcional-integral y derivado, PID para un sistema de control de circuito cerrado de la amplitud de campo aplicada entre dos electrodos. Se evaluó su desempeño en soluciones salinas y en agua pura. Los valores medios del campo a lo largo del tiempo con control PID muestran una tendencia a caer desde el valor inicial de $0,3 \%$. El circuito de control VCA propuesto demostró ser eficaz para mantener los valores de campo a lo largo del tiempo.
\end{abstract}

Palabras clave: Amplificador controlado por voltaje; Controlador derivado integral proporcional; Campo eléctrico alterno; Electroformación; Vesículas unilaminares gigantes.

\title{
1. Introdução
}

A membrana celular forma uma barreira dinâmica e complexa entre a célula viva e seu ambiente mantendo as moléculas no interior da célula. A membrana é responsável pela seletividade e permeabilidade da célula controlando o transporte de nutrientes. O estudo da composição da membrana faz uso de diversas técnicas e metodologias físicas (Dietrich et al., 2001; Lingwood \& Simons, 2010; Sun et al., 2013). Um modelo de sistema bem estabelecido para estudar a estrutura e a dinâmica das membranas são as Vesículas unilamelares gigantes (GUVs). As GUVs consistem em uma bicamada lipídica cheias de fluidos que oferecem um modelo básico e útil de uma célula biológica e apresentam um tamanho comparável com os das células eucarióticas, mimetizando assim o mesmo reservatório lipídico e curvatura da membrana. Por causa disso, as GUVs vêm sendo amplamente aplicadas em pesquisas biológicas como uma membrana biomimética (Eeman \& Deleu, 2010; Fenz \& Sengupta, 2012; Walde et al., 2010). Na literatura existem diversos protocolos de formação de vesículas onde para cada técnica a sua própria desvantagem inerente de acordo com a aplicação. A escolha entre um protocolo específico pode acontecer devido à perícia técnica, a necessidade de equipamentos especializados e o grau em que as características da vesícula precisam ser ajustadas (Bagatolli \& Needham, 2014; Pereno et al., 2017; Politano et al., 2010). A eletroformação para a produção de GUVs é um dos métodos mais prevalentes, pois permite a modulação do processo de hidratação lipídica para formar vesículas relativamente monodispersas e livres de defeitos. O desenvolvimento dessas câmaras de eletroformação vem sendo descritos ao longo dos anos na literatura (Bagatolli \& Needham, 2014; Drabik et al., 2018; Li et al., 2016; Politano et al., 2010), principalmente utilizando campo elétrico de corrente alternada (CA) de baixa frequência, 0,5-1,0 kHz, e uma amplitude de tensão entre 2,0 e 3,0 V aplicada a eletrodos imersos em solução aquosa pura ou soluções salinas.

Ter controle sobre estes importantes aspectos do processo de eletroformação de GUVs pode melhorar o rendimento e a repetibilidade de formação das vesículas. Em diversas aplicações tecnológicas o controle preciso dos campos elétricos aplicados deve ser garantido (Ang et al., 2005a; Ghellab et al., 2019; Ogata, 2009; "The Control Handbook," 2018). Estas aplicações podem estar em processos industriais ou em dispositivos domésticos como tubos de raios catódicos e impressoras laser ou em arranjos experimentais como no processo de eletroformação. Nas aplicações de experimentação biofísica o controle das flutuações de campo induzidas por cargas livres em eletrodos expostos a soluções salinas é essencial. A amplitude CA é relatada como um dos principais fatores a serem controlados durante a eletroformação de GUVs (Bagatolli \& Needham, 2014; Li et al., 2016; Politano et al., 2010). 
Em diversas aplicações tecnológicas o controle preciso dos campos elétricos aplicados deve ser garantido (William S. Levine, 2018) (Katsuhiko Ogata, 2010) (Ang et al., 2005b), estas aplicações podem estar em processos industriais ou em dispositivos domésticos como tubos de raios catódicos e impressoras laser ou em arranjos experimentais como o processo de eletroformação. Nas aplicações de experimentação biofísica o controle das flutuações de campo induzidas por cargas livres em eletrodos expostos a soluções salinas é essencial. A amplitude da corrente alternada (CA) e relatada como um dos principais fatores a serem controlados durante a eletroformação de vesículas gigantes unilamelares (Giant unilamellar vesicles, GUV) (Bagatolli \& Needham, 2014; Li et al., 2016; Politano et al., 2010). O desenvolvimento dessas câmaras de eletroformação vem sendo descritos ao longo dos anos na literatura (Bagatolli \& Needham, 2014; Drabik et al., 2018; Li et al., 2016; Politano et al., 2010), (Santos Junior et al., 2019) e (Santos Junior \& Vieira, 2022) principalmente utilizando campo elétrico CA de baixa frequência, 0,5-1,0 kHz, e uma amplitude de tensão entre 2,0 e 3,0 V aplicada a eletrodos imersos em solução aquosa pura ou soluções salinas. Assim, visando obter um controle sobre este importante aspecto do processo de eletroformação, o objetivo deste trabalho consiste em apresentar um protótipo de circuito para o controle do campo CA.

\section{Metodologia}

Esse sistema de controle que é apresentado pode ser incorporado às câmaras de eletroformação de GUVs para melhorar o rendimento de formação das vesículas. Este protótipo foi desenvolvido com circuitos separados por módulos e utilizando componentes comerciais discretos. Dessa forma foi possível conceber um dispositivo de controle empregando dispositivos eletrônicos de fácil acesso e economicamente acessível em relação às câmaras de eletroformação pré-existentes.

Com base nas proposições descritas projetamos um amplificador controlado por tensão (Voltage-Controlled Amplifier, VCA), que na maioria das vezes é usado como atenuador controlado por voltagem. Ele pode ser encontrado em compressor de áudio ou em circuito limitador de pico. Os circuitos integrados que têm este propósito são de esquemas proprietários feitos pelos detentores das patentes dos mesmos ou empresas licenciadas, isso faz com que o produto tenha um custo elevo. Porém eles podem ser construídos baseados em circuitos modulares separados utilizando componentes comerciais discretos (Self $e t a l$., 2008). Todos os circuitos VCAs disponíveis possuem vantagens e limitações, as mais difíceis de resolver são distorção e a alimentação de tensão de controle. O circuito que foi desenvolvido para este trabalho usa um resistor dependente de luz (Light Dependent Resistor, LDR), outros podem usar diodos, e muitos são controlados por transistores de Efeito de Campo (FET). Nesse projeto, o VCA será utilizado para controlar a amplitude de um sinal, este tipo de comando pode ser configurado usando uma tensão de referência. A tensão de controle deste circuito e obtida por um sinal de modulação por largura de pulso (PulseWidth Modulation, PWM). Este sinal PWM vem do processamento de um microcontrolador ATMEGA328P ${ }^{\circledR}$ (Atmel, San José, Califórnia, EUA), de baixo custo e fácil aplicação, tais como no controle e acionamento de LEDs para estímulo visual em estudos eletro-oculográficos (Junior et al., 2021). Este microcontrolador e responsável por digitalizar o sinal lido nos eletrodos de Platina (Pt) imersos na solução aquosa, fazer os cálculos do algoritmo de controle proporcional-integral e derivativo (ProportionalIntegral-Derivative, PID) e controla o ciclo de trabalho do sinal de saída PWM. Para medir as formas de onda senoidal de tensão AC é usado o padrão da tensão quadrada média da raiz (RMS) que representa o valor da tensão em corrente contínua (CC) equivalente.

\subsection{Oscilador}

$\mathrm{O}$ oscilador gerador do sinal de onda senoidal projetado para uso com este equipamento possui uma frequência de 500 Hz, com uma tensão de saída de 1,0 Vrms. Com níveis de distorção harmônica na faixa de 0,05\% na pureza da forma de onda. Esta frequência e tensão de saída atende os protocolos de eletroformação estudados (Bagatolli \& Needham, 2014; Drabik et al., 
2018; Li et al., 2016; Politano et al., 2010). Este circuito oscilador montado consiste em um núcleo de oscilador de ponte de Wien (Self et al., 2008) e um circuito de estabilização de amplitude. Ele foi montado a partir dos amplificadores operacionais do circuito integrado TL082, que é um amplificador operacional duplo. Alterando os valores de dois resistores da ponte pode-se alterar a frequência do oscilador, garantindo dessa foram a praticidade deste circuito.

\subsection{Controle do LDR}

A combinação de um LDR juntamente com uma fonte de luz rápida, como um diodo emissor de luz (light-emitting diode LED) com um tempo de ativação da ordem de centenas de nanossegundos, apresenta uma distorção relativamente baixa. No entanto, os LDRs são naturalmente lentos, com tempos de resposta normalmente na faixa de 5 milissegundo (ms) a $100 \mathrm{ms,} \mathrm{o}$ que limita a possibilidade de tempos de atuação rápidos. Entretanto, mesmo esse tempo de atuação lento não ofereceu limite para o controle que este circuito é proposto. Um sinal de tensão controla a corrente que aciona o LED para que o brilho total seja definido como nível máximo ou atenuação mínima. O LDR adjacente atua como braço superior ou inferior de um atenuador ativo. Um circuito de controle de corrente linear usando transistores controlada a corrente de acionamento do LED. Este circuito usa a modulação por largura de pulso (Pulse-Width Modulation PWM) para controlar a corrente máxima ou brilho máximo. O isolamento intrínseco do circuito devido a sua separação física torna o arranjo LED / LDR uma conexão de controle opticamente isolada.

\subsection{Controle do LED}

Os LEDs têm uma voltagem mínima para o acionamento, o utilizado possui uma tensão mínima de 2,4 V, e se iluminam rapidamente, a partir da voltagem mínima, a latências de acionamento está em torno de nanossegundos. Seu brilho é aumentado ligeiramente quando são adicionadas pequenas voltagens variando até a máxima tensão de cerca de 2,7 V. É muito melhor controlar o brilho do LED usando variação de corrente, não de voltagem, pois são materiais não ôhmicos com a relação entre a corrente e a tensão não linear. Para isso, precisamos transformar a voltagem em corrente utilizando um circuito que emprega um transistor de efeito de campo metal óxido semicondutora (Metal Oxide Semiconductor Field Effect Transistor MOSFET). O transistor trabalha em sua região de controle linear, e é usado como conversor de sinal de tensão para corrente. O Transistor MOSFET é controlado por dois transistores um NPN e um PNP. O transistor PNP tem sua base polariza para a terra e conectada ao sinal PWM. O trabalho do transistor NPN e forneça o desligamento do Transistor MOSFET caso a corrente do circuito ultrapasse um valor máximo de cerca de $25 \mathrm{~mA}$. Desta forma, um sinal PWM pode ser usado para variar o brilho de uma carga de LED com alguns miliamperes de corrente.

\subsection{Atenuador}

O LED está apontando diretamente para um LDR, sendo o conjunto envolto por tubos termo retrátil para que nenhuma luz externa entre. Quanto maior o brilho do LED, menor será a resistência do LDR. Essa combinação nos dá um resistor variável, responsável pela variação de ganho do circuito de um amplificador inversor (AI). Esse amplificador é montado empregando um dos amplificadores operacionais do circuito integrado TL074, que é um amplificador operacional quádruplo. Este circuito de variação de ganho é montado entre dois filtros passa baixa, o primeiro com uma frequência de corte de cerca de $1,5 \mathrm{kHz}$ e o segundo com cerca de $1 \mathrm{kHz}$. Após o controle de ganho e a última etapa de filtragem o sinal segue para a etapa amplificadora de potência com ganho unitário.

\subsection{Conversor CA para CC}

O circuito conversor CA para CC projetado possui precisão de conversão menor que $1 \%$, com menos de $1 \%$ de ondulação. Para evitar que as possíveis variações de impedância do sinal de entrada afetem a conversão CC este circuito possui um 
amplificador diferencial na entrada de sinal, garantindo que a etapa de conversão seja acionada por uma impedância baixa, evitando erros de conversão que esse fato pudesse causar. A etapa de conversão possui um controle de deslocamento e um retificador de precisão de onda completa. O conversor de onda completa possui um ganho de voltagem de 1,11 para fornecer a correção do fator de forma, com uma etapa de integração, possuindo uma saída de baixa impedância. O circuito conversor e calibrado para que 1,0 V RMS seja igual a $0,707 \mathrm{~V}$ de pico. Para montar esse conversor foi utilizado o circuito integrado TL074 que apresenta uma linearidade satisfatória para a frequência do sinal de onda senoidal usado no equipamento.

A Figura 1 ilustra o protótipo da placa em circuito impresso do oscilador, Figura 1(a), do atenuador, Figura 1(b), e do conversor AC para CC, Figura 1(c).

Figura 1. Imagem detalhando os componentes eletrônicos e conexões externas da placa do oscilador, painel (a) e da placa do atenuador, painel (b) e da placa do conversor RMS para CC, painel (c).

(a)

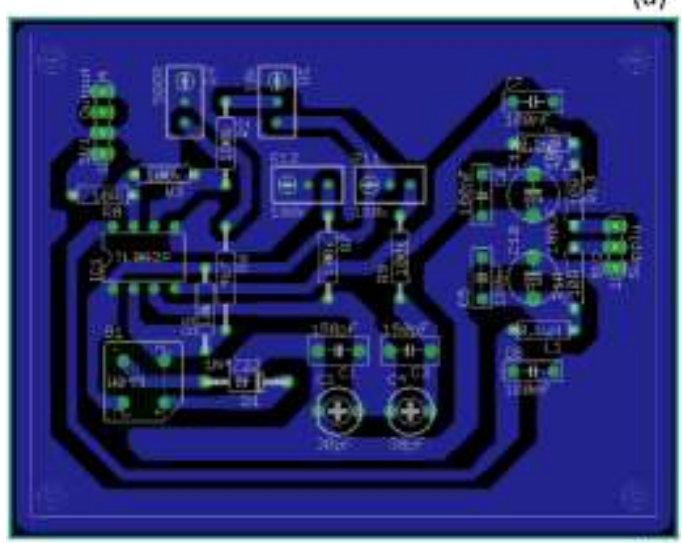

(b)

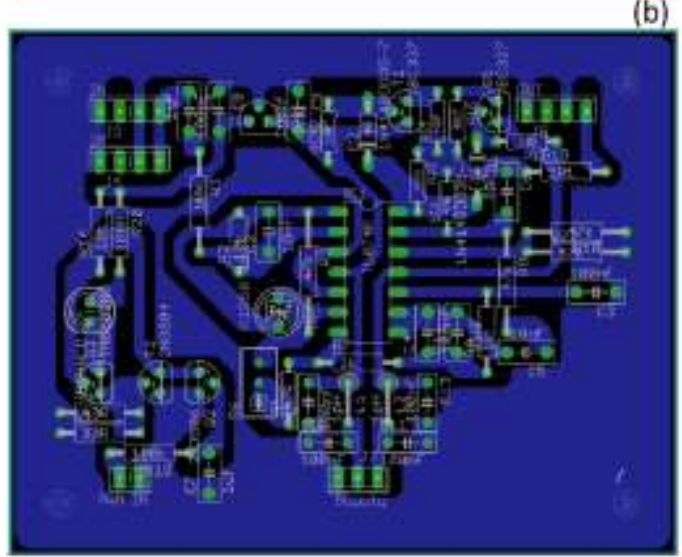

(C)

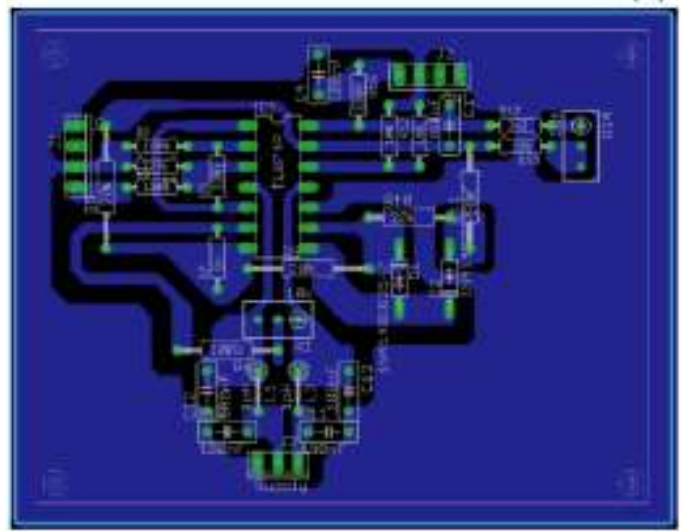

Fonte: Autores. 


\subsection{Algoritmo de Controle}

O sinal de onda senoidal vindo do controlador VCA está conectado nos eletrodos de platina imersos na solução aquosa. Estes eletrodos imersos na solução aquosa também estão conectados no conversor de corrente alternada CA para corrente contínua CC (conversor true RMS-DC). Este sinal CC serve de parâmetro de entrada para calcular a resposta de saída do algoritmo PID, esse processo de controle em malha fechada tenta levar o sinal de entrada o mais próximo do valor desejado. O diagrama do processo de funcionamento do controle pode ser visto na Figura 2(a).

O processamento do algoritmo PID deste trabalho tem como resposta de saída o controle de 255 pontos do ciclo de trabalho de um sinal PWM. O controle dos pontos do ciclo de trabalho corresponde a uma variação de largura de pulso de 0 a 100\%. Assim, para o circuito atenuador VCA, 0 seria atenuação máxima e 100\% atenuação mínima. O diagrama do processo de funcionamento do controle PID pode ser visto na Figura 2(b). Para verificar os efeitos do ajuste desse sistema de controle, três amostras diferentes foram testadas. Uma solução de água pura, uma solução de tampão fosfato-salino (phosphate buffered saline PBS) contendo $150 \mathrm{mM}$ de $\mathrm{NaCl}$ e outra solução de PBS contendo $600 \mathrm{mM}$ de $\mathrm{NaCl}$.

Figura 2. Representação em diagrama de blocos do sistema de controle da câmara de eletroformação. (a) diagrama do processo de funcionamento da câmara, (b) diagrama do processo de funcionamento do controle PID.

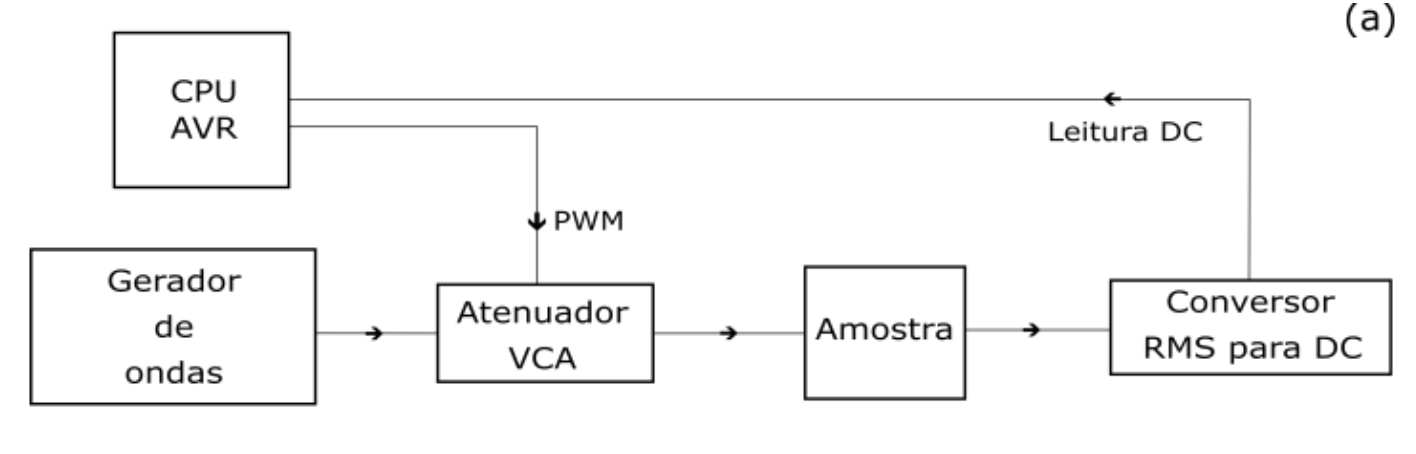

(b)

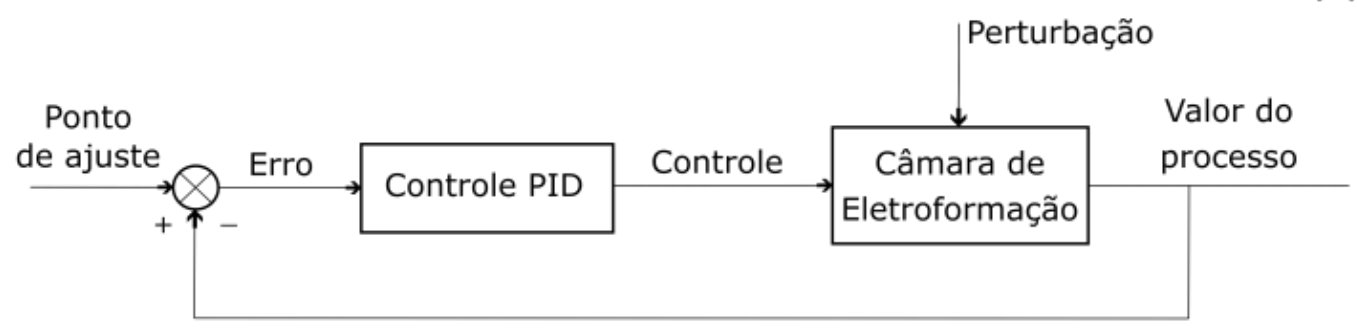

Fonte. Autores.

O método de controle PID se caracteriza por uma combinação de técnicas de controle proporcional, integral e derivativo. No entanto, o algoritmo PID requer uma determinação previa dos ganhos proporcionais (Kp), integrais (Ki) e derivados (Kd). Essas constantes estão associadas às variáveis que devem ser controladas. Assim, um algoritmo de autoajuste baseado no acúmulo dos valores destes ganhos foi usado para minimizar os erros destas contates. O processo de autoajuste das constantes foi realizado usando uma solução PBS contendo $150 \mathrm{mM}$ de $\mathrm{NaCl}$ com um ponto de ajuste de $400 \mathrm{mVrms}$. Após a obtenção dos valores de Kp, Ki e Kp, o algoritmo de controle PID foi testado nos três tipos de soluções aquosas, para manter um ponto de ajuste de $600 \mathrm{mV}$ RMS. 


\subsection{Protocolo experimental}

Os dados foram coletados a partir de eletrodos de platina submersos em $525 \mu \mathrm{L}$ de solução aquosa. O arranjo onde os eletrodos são presos e a amostra aquosa e depositada e feitos de politetrafluoroetileno (PTFE), material este escolhido pelo seu caráter muito pouco reativo. A aquisição de dados foi realizada durante 25 min. e os valores do campo CA foram coletados a cada segundo.

\section{Resultados e Discussão}

A distorção de um LDR depende da corrente que atravessa o dispositivo, assim para manter a distorção baixa a corrente deve ser mínima. A distorção medida no LDR para uma voltagem de entrada de 2,5 V RMS sob uma voltagem de 2,0 V RMS, chegou a $0,12 \%$. Para efetuar esta medida foi utilizado um divisor de tensão feito com um resistor em serie com o LDR. A tensão de entrada que será utilizada é da ordem de 1,0 V RMS, portanto com uma corrente mais baixa, a distorção terá um valor abaixo de $0,12 \%$, independentemente da atenuação.

A Figura 3 mostra o comportamento dos valores de campo CA medidos em eletrodos de Pt imersos em água pura, solução de PBS com 150 e 600 mM de NaCl, respectivamente. Os valores efetivos do campo CA obtidos para as três soluções aquosas mostram uma tendência de controle estável em comparação com o valor de ajuste, no intervalo de 25 minutos, denotando que o algoritmo de controle PID usado, mantém o valor satisfatoriamente próximo do ponto de ajuste. Observe que para os diferentes tipos de solução, que representa diferentes tipos de carga para o circuito atenuador, a resolução do controle de atenuação da combinação LED / LDR e o intervalo de controle do PWM se mostrou eficaz para fornecer a alteração necessária para manter o valor do campo CA próxima ao ponto de ajuste.

Como pode ser visto o controle PID realizado pelo microcontrolador AT-mega328p ${ }^{\circledR}$ utilizando os parâmetros descritos na seção Métodos, mostrou-se muito eficaz. Estabilizando em alguns segundos as oscilações mais intensas que pode ser vista nos segundos iniciais de cada curva na Figura 3(a), (b) e (c).

A resolução do circuito de controle VCA controlado pelo algoritmo PID pode garantir um controle de amplitude do sinal CA garantido um desvio padrão menor que $2 \mathrm{mVrms}$ para as diferentes soluções aquosas para o ponto de ajuste de 600 mVrms. Uma comparação dos valores estatísticos obtidos para o ponto de ajuste de $600 \mathrm{mVrms}$ da amplitude do campo CA é apresentada na Tabela 1, onde $\mathrm{N}$ representa o número de interações; Média, e o valor médio aritmético; SD, o desvio padrão; Min, o valor mínimo, e Max, o valor máximo em mV.

Tabela 1. Parâmetros de tensão AC obtidos para eletrodos de Platina imersos em diferentes soluções aquosas.

\begin{tabular}{llllll}
\hline Amostra & N & Media & SD & Min & Max \\
\hline Solução aquosa & 1471 & 599.99 & 1.47 & 595.13 & 605.82 \\
$\mathrm{NaCl} 150 \mathrm{mM}$ & 1472 & 599.29 & 1.54 & 593.57 & 603.58 \\
$\mathrm{NaCl} 600 \mathrm{mM}$ & 1467 & 599.45 & 1.37 & 595.01 & 603.06 \\
\hline
\end{tabular}

Nota: $\mathrm{N}$ representa o número de amostras; Média e o valor médio aritmético; SD, o desvio padrão; Min, o valor mínimo e Max, o valor máximo em milivolt. Fonte. Autores.

O protótipo de controle do campo CA pode além de controlar o experimento automaticamente ser uma alternativa viável, pois apresenta um baixo custo financeiro para sua aquisição, pouco mais de 70 dólares americanos. O custo total do protótipo juntamente com o preço de alguns componentes principais para sua fabricação pode ser visto na Tabela 2. 
Tabela 2. Custo dos principais componentes eletrônicos incluindo o custo somado dos componentes restantes.

\begin{tabular}{lccc}
\hline Componente & Quantidade & Custo por unidade & Custo total \\
\hline Trimpots & 07 & 2.60 & 18.20 \\
Resistores & 42 & 0.10 & 4.20 \\
Transistors & 05 & 0.40 & 2.00 \\
Diodos & 05 & 0.10 & 0.50 \\
Circuitos integrados & 03 & 1.07 & 3.21 \\
Capacitores & 22 & 0.43 & 9.46 \\
Modulo ATmega328p & 01 & 19.00 & 19.00 \\
Diversos & 01 & 15.00 & 15.00 \\
\hline
\end{tabular}

Total: 71.57

Nota: Custo em dólares americanos dos principais componentes eletrônicos para a montagem do protótipo de controle de campo CA. Fonte. Autores.

Figura 3. Comportamento dos valores do campo CA medidos em eletrodos de Pt imersos em (A) solução aquosa; (B) PBS$\mathrm{NaCl150} \mathrm{mM}$ e (C) PBS-NaCl 600 mM. A linha vermelha indica o valor do ponto de ajuste do algoritmo PID.
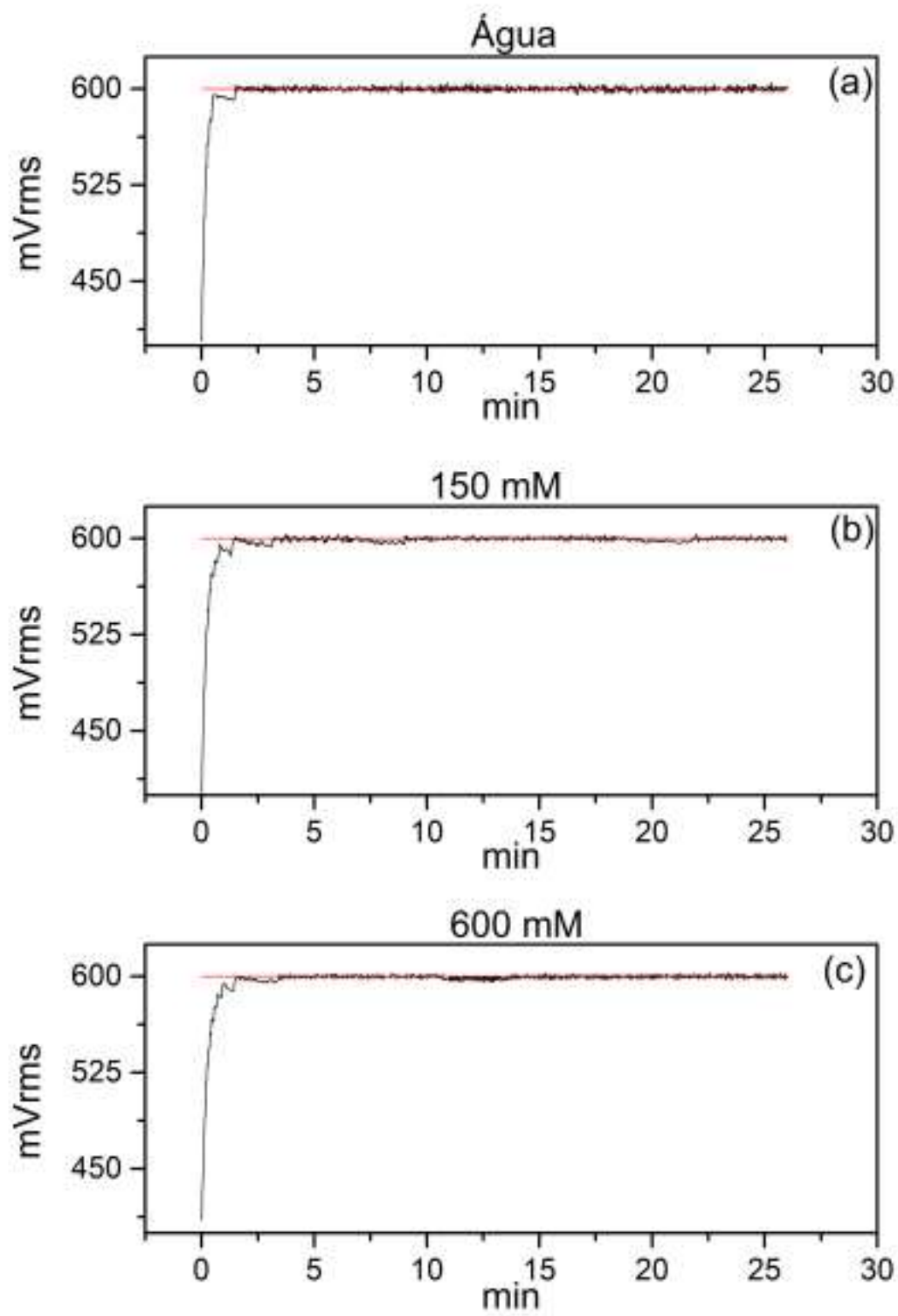

Fonte. Autores. 
O protótipo desenvolvido pode possibilitar futuros estudos sobre diferentes protocolas de eletroformação de GUVs explorando as vantagens e desvantagens de diferentes rampas de controle tanto na amplitude de campo quanto na temperatura. No entanto, uma comparação entre diferentes protocolos de eletroformação vai além das perspectivas deste trabalho, mas poderá ser abordada em um trabalho posterior. É importante ressaltar que o protótipo do sistema automatizado também permite uma variação controlada e gradativa da temperatura de eletroformação das GUVs. Seria útil na eletroformação de GUVs que apresentam domínios lipídicos devido à segregação de fase lateral, uma vez que este tipo de vesículas requer um tratamento térmico específico durante e após sua formação (Angelova \& Dimitrov, 1986), (Bagatolli \& Gratton, 2000a, 2000b), (Busto et al., 2009), (Mollinedo \& Gajate, 2015) e (Reeves \& Dowben, 1969). Assim, abre-se uma janela para explorar experimentalmente a correlação entre aspectos fundamentais da eletroformação AC de vesículas lipídicas gigantes.

\section{Conclusão}

O método de controle por atenuação de um sinal de entrada para controlar a amplitude do campo CA aplicado em eletrodos de Platina imersos em solução aquosa mostrou ter bastante eficácia. Utilizando o circuito atenuador VCA baseado na combinação LED / LDR, foi possível controlar a amplitude do campo CA aplicada nos eletrodos e o controle das flutuações de campo, sendo este um dos principais fatores a serem controlados durante a eletroformação descritas na literatura (Bagatolli \& Needham, 2014; Drabik et al., 2018; Li et al., 2016; Politano et al., 2010). O tempo de resposta lento para a variação da resistência do LDR, na faixa de 5 milissegundo, não afetou o processo de controle do campo CA, pois como pode ser visto na figura 3, as variações iniciais que ocorreram têm respostas da ordem de segundos, que ao longo do tempo de execução do controle foram controladas. O protocolo PID mostrou-se muito eficaz na manutenção dos valores dos pontos de ajustes para o campo CA dos eletrodos usando o ciclo de trabalho do sinal PWM para controlar o valor da atenuação. Com esse sistema de controle proposto e possível obter um desvio de menos de $2 \mathrm{mV}$ RMS para um valor de ajuste de $600 \mathrm{mV}$ RMS, correspondendo a um erro de cerca de $0,3 \%$ do valor desejado. Sendo assim o sistema de controle desenvolvido uma alternativa barata e eficaz, pois custando menos de 80 dólares americanos foi feito o controle do processo de eletroformação de vesículas unilamelares gigantes.

\section{Agradecimentos}

Os autores agradecem a Universidade Federal de Goiás (UFG) pela infraestrutura disponibilizada, e ao apoio financeiro das agências brasileiras de fomento à pesquisa: Conselho Nacional de Desenvolvimento Científico e Tecnológico (CNPq) e a Fundação de Amparo à Pesquisa do Estado de Goiás (FAPEG).

\section{Referências}

Ang, K. H., Chong, G., \& Li, Y. (2005a). PID control system analysis, design, and technology. IEEE Transactions on Control Systems Technology, 13(4). https://doi.org/10.1109/TCST.2005.847331

Ang, K. H., Chong, G., \& Li, Y. (2005b). PID control system analysis, design, and technology. IEEE Transactions on Control Systems Technology, 13(4), 559576. https://doi.org/10.1109/TCST.2005.847331

Angelova, M. I., \& Dimitrov, D. S. (1986). Liposome electroformation. Faraday Discussions of the Chemical Society, 81, 303-311. https://doi.org/10.1039/DC9868100303

Bagatolli, L. A., \& Gratton, E. (2000a). Two photon fluorescence microscopy of coexisting lipid domains in giant unilamellar vesicles of binary phospholipid mixtures. Biophysical Journal, 78(1), 290-305. https://doi.org/10.1016/S0006-3495(00)76592-1

Bagatolli, L. A., \& Gratton, E. (2000b). A Correlation between Lipid Domain Shape and Binary Phospholipid Mixture Composition in Free Standing Bilayers: A Two-Photon Fluorescence Microscopy Study. Biophysical Journal, 79(1), 434-447. https://doi.org/10.1016/S0006-3495(00)76305-3

Bagatolli, L. A., \& Needham, D. (2014). Quantitative optical microscopy and micromanipulation studies on the lipid bilayer membranes of giant unilamellar vesicles. In Chemistry and Physics of Lipids (Vol. 181). https://doi.org/10.1016/j.chemphyslip.2014.02.009 
Research, Society and Development, v. 11, n. 1, e44711124802, 2022

(CC BY 4.0) | ISSN 2525-3409 | DOI: http://dx.doi.org/10.33448/rsd-v11i1.24802

Busto, J. v, Fanani, M. L., de Tullio, L., Sot, J., Maggio, B., Goñi, F. M., \& Alonso, A. (2009). Coexistence of immiscible mixtures of palmitoylsphingomyelin and palmitoylceramide in monolayers and bilayers. Biophysical Journal, 97(10), 2717-2726. https://doi.org/10.1016/j.bpj.2009.08.040

Dietrich, C., Bagatolli, L. A., Volovyk, Z. N., Thompson, N. L., Levi, M., Jacobson, K., \& Gratton, E. (2001). Lipid Rafts Reconstituted in Model Membranes. Biophysical Journal, 80(3), 1417-1428. https://doi.org/10.1016/S0006-3495(01)76114-0

Drabik, D., Doskocz, J., \& Przybyło, M. (2018). Effects of electroformation protocol parameters on quality of homogeneous GUV populations. Chemistry and Physics of Lipids, 212. https://doi.org/10.1016/j.chemphyslip.2018.01.001

Eeman, M., \& Deleu, M. (2010). From biological membranes to biomimetic model membranes. Biotechnologie, Agronomie, Société et Environnement, 14(4), 719-736.

Fenz, S. F., \& Sengupta, K. (2012). Giant vesicles as cell models. Integrative Biology (United Kingdom), 4(9), 982-995. https://doi.org/10.1039/c2ib00188h

Ghellab, S. E., Mu, W., Li, Q., \& Han, X. (2019). Prediction of the size of electroformed giant unilamellar vesicle using response surface methodology. Biophysical Chemistry, 253(July), 106217. https://doi.org/10.1016/j.bpc.2019.106217

Junior, G. de M., Vieira, S. L., Furriel, G. P., Filho, R. M. de O., Silva, B. C. R., \& Naves, J. S. (2021). Pré-processamento de sinais Eletro-oculográficos (EOG). Research, Society and Development, 10(4), e28410414221. https://doi.org/10.33448/rsd-v10i4.14221

Katsuhiko Ogata. (2010). Modern Control Engineering, (5th ed.), Pearson. https://www.pearson.com/us/higher-education/program/Ogata-Modern-ControlEngineering-5th-Edition/PGM100186.html

Li, Q., Wang, X., Ma, S., Zhang, Y., \& Han, X. (2016). Electroformation of giant unilamellar vesicles in saline solution. Colloids and Surfaces B: Biointerfaces, 147. https://doi.org/10.1016/j.colsurfb.2016.08.018

Lingwood, D., \& Simons, K. (2010). Lipid Rafts As a Membrane-Organizing Principle. Science, 327(5961), 46-50. https://doi.org/10.1126/science.1174621

Mollinedo, F., \& Gajate, C. (2015). Lipid rafts as major platforms for signaling regulation in cancer. Advances in Biological Regulation, 57, 130-146. https://doi.org/10.1016/j.jbior.2014.10.003

Ogata, K. (2009). Modern Control Engineering Fifth Edition. In IEEE Transactions on Automatic Control. 17(3).

Pereno, V., Carugo, D., Bau, L., Sezgin, E., Bernardino de la Serna, J., Eggeling, C., \& Stride, E. (2017). Electroformation of Giant Unilamellar Vesicles on Stainless Steel Electrodes. ACS Omega, 2(3), 994-1002. https://doi.org/10.1021/acsomega.6b00395

Politano, T. J., Froude, V. E., Jing, B., \& Zhu, Y. (2010). AC-electric field dependent electroformation of giant lipid vesicles. Colloids and Surfaces B: Biointerfaces, 79(1), 75-82. https://doi.org/10.1016/j.colsurfb.2010.03.032

Reeves, J. P., \& Dowben, R. M. (1969). Formation and properties of thin-walled phospholipid vesicles. Journal of Cellular Physiology, 73(1), 49-60. https://doi.org/10.1002/jcp.1040730108

Santos Junior, J. L. dos, Mendanha, S. A., Vieira, S. L., \& Goncalves, C. (2019). Portable Proportional-Integral-Derivative controlled chambers for giant unilamellar vesicles electroformation. Biomedical Physics and Engineering Express, 5(4), 47002. https://doi.org/10.1088/2057-1976/ab1a1b

Santos Junior, J. L. dos, \& Vieira, S. L. (2022). Design of an Alternating Current Field Controller for Electrodes Exposed to Saline Solutions. In Teodiano Freire Bastos-Filho, Eliete Maria de Oliveira Caldeira, \& Anselmo Frizera-Neto (Eds.), Proceedings of Brazilian Congress on Biomedical Engineering 2020, October 26-30, 2020, Vitória, Brazil (27, 881-885). Springer International Publishing.

Self, D., Sinclair, I., Nathan, J., \& Duncan, B. (2008). Audio Engineering: Know It All. 907. http://books.google.com/books?id=zKsrPQAACAAJ\&printsec=frontcover

Sun, T. L., Sun, Y., Lee, C. C., \& Huang, H. W. (2013). Membrane permeability of hydrocarbon-cross-linked peptides. Biophysical Journal, 104(9), 1923-1932. https://doi.org/10.1016/j.bpj.2013.03.039

The Control Handbook. (2018). In The Control Handbook. CRC Press. https://doi.org/10.1201/b10382

Walde, P., Cosentino, K., Engel, H., \& Stano, P. (2010). Giant Vesicles: Preparations and Applications. ChemBioChem, 11(7), 848-865. https://doi.org/10.1002/cbic.201000010

William S. Levine. (2018). The Control Handbook (three volume set). In The Control Handbook: (2nd ed.), CRC Press. https://doi.org/10.1201/9781315218694

Clique ou toque aqui para inserir o texto. 